\title{
Concurrent Map Building and Localization with Landmark Validation
}

\author{
Juan Andrade-Cetto and Alberto Sanfeliu \\ Institut de Robòtica i Informàtica Industrial, UPC-CSIC \\ Llorens i Artigas 4-6, 08034 Barcelona, Spain \\ cetto, sanfeliuliri.upc.es
}

\begin{abstract}
This communication addresses the issue of concurrent map building and localization (CML) for a mobile robot in an unknown environment. The proposed solution extends over previous contributions in that the environment must not be static, nor the landmarks be uniquely identifiable. To this aim we introduce a map model that includes not only the robot and landmark locations in a reference frame, but also a model for landmark quality assessment. Convergence of the map covariance is preserved in the new map model.
\end{abstract}

Keywords: map building, mobile robot navigation, CML, SLAM.

\section{Introduction}

The use of stochastic models for map building and localization in mobile robotics has gained much popularity in recent years $[3,5,9]$. Of particular interest is the use of predictive filters to estimate the robot position and uncertainty, and to update these estimates from sensor readings while at the same time building an incremental map of the environment from observations [1, 2, 4, 6].

One of the most critical limitations to the application of such estimation-theoretic approaches to CML is the data association problem. Data association refers to the issue of matching an observation with a previously learned entity in the environment. Some techniques can be used to alleviate the data association problem, such as search space reduction, or landmark invariance characterization. Obviously there is always a compromise between the possibility of full invariant landmark characterization and the difficulty to extract such characterizing features from raw sensor data.

Nevertheless, as we address issues such as viewpoint invariance or feature extraction from sensor data, it is overwhelming how undesired environment dynamics, occlusions, and sensor noise can still make data association a daunting task. One possibility to overcome these difficulties is the deployment of man-made beacons to aid in localization. Unfortunately, there exist multiple situations when this is not possible, and a map must be constructed without environment contamination. An alternative explored in this article is the inclusion of temporal and spatial landmark quality measures into the map model.

In [4] for example, landmark robustness is addressed as an implementation detail only, suggesting a quality measure based on the probability density function of the observations associated to a given landmark, disregarding the temporal dispersion of such observations.

In [7] the problem of temporal dispersion of observations is analyzed in the context of landmark initialization. A revised implementation of the CML algorithm is presented where the robot pose at each time instance is added to the map state vector until all landmark measurements from that given step had been either processed or discarded. The idea suggests that the combination of observations of the same landmark from various viewpoints aids in assessing the robustness of such landmark. However, this methodology not only increases the computational burden of the CML algorithm, but diverges from the fundamental motivation of using a Kalman filter to maintain a parametric representation of the history of the uncertainty of robot and landmark localization by means of an estimated full-covariance matrix.

On the contrary, we propose an extended map model that includes the pose of the robot and the location of the landmarks in the map, and a set of landmark quality states. The advantages offered by the possibility of landmark quality assessment allow the pruning of the map state at each iteration in terms of both spatial and temporal landmark quality, thus reducing the impact of false data association. In the proposed approach, the size of the state vector does not depend on the number of iterations. Moreover, it is still possible to achieve a monotonically decreasing map covariance matrix.

\section{Map model}

In the typical full-covariance KF-based CML the state vector is composed of the position of the robot $\mathbf{x}_{r}$ and the map features $\mathbf{x}_{f_{i}}$ [9]. Consider an extension to this model by appending landmark quality states $\mathcal{X}_{f_{i}}$.

$$
\mathbf{x}=\left[\mathbf{x}_{r}^{\top}, \mathbf{x}_{f_{1}}^{\top}, \ldots, \mathbf{x}_{f_{N}}^{\top}, \mathcal{X}_{f_{1}}^{\top}, \ldots, \mathcal{X}_{f_{N}}^{\top}\right]^{\top}
$$

The motion of the robot is governed by the discrete-time 
state transition model

$$
\begin{aligned}
\mathbf{x}(k) & =\mathbf{f}(\mathbf{x}(k-1), \mathbf{u}(k), \mathbf{v}(k)) \\
\mathbf{z}_{i}(k) & =\mathbf{h}_{i}\left(\mathbf{x}(k), \mathbf{w}_{i}(k)\right) \quad \forall i
\end{aligned}
$$

where the row elements in Eq. 2 take the form

$$
\begin{aligned}
\mathbf{f}_{r}(k) & =\mathbf{x}_{r}(k-1)+\mathbf{u}_{r}(k)+\mathbf{v}_{r}(k) \\
\mathbf{f}_{f_{i}}(k) & =\mathbf{x}_{f_{1}}(k-1) \\
\mathbf{f}_{\mathcal{X}_{i}}(k) & =\mathbf{f}_{\mathcal{X}_{i}}\left(\mathcal{X}_{f_{i}}(k-1), \mathbf{u}_{f_{i}}(k), \mathbf{v}_{f_{i}}(k)\right)
\end{aligned}
$$

The term $\mathbf{f}_{r}(k)$ represents the robot dynamics, $\mathbf{u}_{r}(k)$ is the vehicle control command, $\mathbf{v}_{r}(k)$ includes unmodeled robot dynamics and noise with zero mean and covariance matrix $\mathbf{Q}_{r}(k), \mathbf{u}_{f_{i}}(k)$ is a landmark identification stamp with zero mean data association uncertainty $\mathbf{v}_{f_{i}}(k)$ and covariance matrix $\mathbf{Q}_{f_{i}}(k)$. The nonlinear observation model $\mathbf{z}_{i}(k)$ for the $i$-th landmark at time step $k$ is expressed as a combination of the robot pose and the location of such map feature, with $\mathbf{w}_{i}(k)$ the measurement noise with zero mean and covariance matrix $\mathbf{S}_{i}(k)$.

The possibly nonlinear expression in Eq. 6 models the proposed landmark quality. In the following sections we describe a candidate function to model this landmark quality, and rework the formulation of the full-covariance Extended Kalman Filter approach to CML. Convergence of the map covariance is also shown.

\section{Landmark temporal uncertainty}

One possibility in the selection of $\mathbf{f}_{\mathcal{X}_{i}}(k)$ is to have an exponential decay rule for the computation of the landmark temporal quality. In this way, each landmark in the map will have an associated memory cell to register how persistent, and how old that landmark is. If a landmark can only be tracked over sensor data for a short period of time, its quality measure will decay, indicating the map building algorithm that it should not be considered a relevant feature for robot localization. Moreover, the map state vector could be pruned if the value of the landmark quality state falls below some criteria.

Thus, the nonlinear update rule in Eq. 6 can be of the form

$$
\mathcal{X}_{f_{i}}(k)=\frac{1}{1+e^{-\left(\alpha\left(u_{f_{i}}(k)+v_{f_{i}}(k)\right)+\beta \mathcal{X}_{f_{i}}(k-1)\right)}}
$$

with $u_{f_{i}}(k)$ a new input to the system, i.e., a landmark identification stamp

$$
u_{f_{i}}(k)=\left\{\begin{array}{cll}
0 & : & \text { unobserved landmark } \\
1 & : & \text { observed landmark }
\end{array}\right.
$$

and $\alpha$ an input weight used to regulate the contribution of such landmark identification over the previous map configuration, $\beta$ a memory weight used to regulate the contribution of the previous landmark quality state over its new value, and $v_{f_{i}}(k)$ zero mean Gaussian noise indicating the probability of landmark mismatch.

\section{Prediction}

To predict the location of the robot and the state of the map purely from motion commands and landmark match stamps one can compute a noise free estimate of an a priori approximation to the map model in Eq. 2 with

$$
\mathbf{x}(k \mid k-1)=\mathbf{f}(\mathbf{x}(k-1 \mid k-1), \mathbf{u}(k), 0)
$$

And, as the mobile robot is commanded, the uncertainty of its location and that of the map features is incremented. An a priori estimate to the map state covariance matrix showing this increase of uncertainty can be computed with

$$
\begin{gathered}
\mathbf{P}(k \mid k-1)=\begin{array}{l}
\mathbf{F}_{\mathbf{x}}(k) \mathbf{P}(k-1 \mid k-1) \mathbf{F}_{\mathbf{x}}(k)^{\top}+ \\
\\
\mathbf{F}_{\mathbf{v}}(k) \mathbf{Q}(k) \mathbf{F}_{\mathbf{v}}(k)^{\top}
\end{array} \\
\mathbf{F}_{\mathbf{x}}(k)=\left[\begin{array}{lll}
\mathbf{I} & & \\
& \mathbf{I} & \\
& & \nabla_{\mathcal{X}} \mathbf{f}_{\mathcal{X}}
\end{array}\right], \quad \mathbf{F}_{\mathbf{v}}(k)=\left[\begin{array}{lll}
\mathbf{I} & & \\
& \mathbf{0} & \\
& & \nabla_{\mathbf{v}} \mathbf{f}_{\mathcal{X}}
\end{array}\right]
\end{gathered}
$$

For the case when $\mathbf{f}_{\mathcal{X}_{i}}$ is chosen as in Eq. 6, the diagonal entries on the partial derivatives in Eq. 11 take the form

$$
\begin{aligned}
\nabla_{\mathbf{x}} \mathbf{f}_{\mathcal{X}_{i}} & =\frac{-\beta e^{-\left(\alpha u_{f_{i}}(k)+\beta \mathcal{X}_{f_{i}}(k-1 \mid k-1)\right)}}{\left(1+e^{-\left(\alpha u_{f_{i}}(k)+\beta \mathcal{X}_{f_{i}}(k-1 \mid k-1)\right)}\right)^{2}} \\
\nabla_{\mathbf{v}} \mathbf{f}_{\mathcal{X}_{i}} & =\frac{-\alpha e^{-\left(\alpha u_{f_{i}}(k)+\beta \mathcal{X}_{f_{i}}(k-1 \mid k-1)\right)}}{\left(1+e^{-\left(\alpha u_{f_{i}}(k)+\beta \mathcal{X}_{f_{i}}(k-1 \mid k-1)\right)}\right)^{2}}
\end{aligned}
$$

\section{Correction}

Once the observation $\mathbf{z}_{i}(k)$ of a landmark coming from sensor data has been matched to its corresponding map item, the estimate computed in Eq. 9 can be revised by adding a correction term

$$
\mathbf{x}(k \mid k)=\mathbf{x}(k \mid k-1)+\mathbf{K}_{i}(k) \mathbf{e}_{i}(k)
$$

with measurement error

$$
\mathbf{e}_{i}(k)=\mathbf{z}_{i}(k)-\mathbf{h}_{i}(\mathbf{x}(k \mid k-1), 0)
$$

The Kalman gain $\mathbf{K}_{i}(k)$ is computed with

$$
\begin{aligned}
& \mathbf{K}_{i}(k)=\mathbf{P}(k \mid k-1) \mathbf{H}_{i}(k)^{\top} \times \\
& \quad\left(\mathbf{H}_{i}(k) \mathbf{P}(k \mid k-1) \mathbf{H}_{i}(k)^{\top}+\mathbf{S}_{i}(k)\right)^{-1}
\end{aligned}
$$


with $\mathbf{H}_{i}(k)$ the observation Jacobian, comprised of the robot and landmark observation Jacobians

$$
\begin{array}{r}
\mathbf{H}_{i}(k)=\left[\nabla_{r} \mathbf{h}_{i}(k), \mathbf{0}, \ldots, \mathbf{0}, \nabla_{f_{i}} \mathbf{h}_{i}(k), \mathbf{0}, \ldots, \mathbf{0}\right. \\
\left.\mathbf{0}, \ldots, \mathbf{0}, \nabla_{\mathcal{X}_{i}} \mathbf{h}_{i}(k), \mathbf{0}, \ldots, \mathbf{0}\right]
\end{array}
$$

Finally, the a posteriori estimate of the map state error covariance can also be revised once a measurement has taken place. It is computed with

$$
\mathbf{P}(k \mid k)=\left(\mathbf{I}-\mathbf{K}_{i}(k) \mathbf{H}_{i}(k)\right) \mathbf{P}(k \mid k-1)
$$

Note that the incomplete measurement provided by each matched landmark contributes only to the revision of the map states directly associated to that particular landmark. By successively computing Eqs. 14-18 for all matched landmarks the constraints of the system are met regaining observability [10].

\section{Convergence of the map covariance matrix}

It has been shown in [8] how in the original KF-based CML formulation the map state covariance submatrix associated with the landmark estimates decreases monotonically as successive observations are made. We show here how this result also extends in our revised map model. The key element in this analysis is the fact that no process noise is associated to the position of the landmarks in the environment, but only to their proper identification, i.e., data association mismatches.

Writing the state covariance matrix in block form

$$
\mathbf{P}=\left[\begin{array}{ccc}
\mathbf{P}_{r} & \mathbf{P}_{r f} & \mathbf{P}_{r \mathcal{X}} \\
\mathbf{P}_{r f}^{\top} & \mathbf{P}_{f} & \mathbf{P}_{f \mathcal{X}} \\
\mathbf{P}_{r \mathcal{X}}^{\top} & \mathbf{P}_{f \mathcal{X}}^{\top} & \mathbf{P}_{\mathcal{X}}
\end{array}\right]
$$

we want to prove the following theorem.

Theorem 1. The determinant of the map covariance matrix decreases monotonically as successive observations are made.

Proof. The proof benefits from the following three properties of positive semi-definite ( $p s d$ ) matrices: a) if $\mathbf{A}$ is $p s d$ then $\mathbf{B A B} \mathbf{B}^{\top}$ is also $p s d$; if $\mathbf{B}$ is also $p s d$ then b) $\mathbf{A}+\mathbf{B}$ is psd, and c) $\operatorname{det} \mathbf{A} \leq \operatorname{det}(\mathbf{A}+\mathbf{B})$.

Given an initialization of $\mathbf{P}(0 \mid 0)$ with a $p s d$ matrix, and that $\mathbf{Q}(k)$ and $\mathbf{S}(k)$ are both also $p s d$; from the three properties cited above, and by induction from Eqs. 10 and 18, $\mathbf{P}(k \mid k-1), \mathbf{P}(k \mid k)$, and $\mathbf{K}_{i}(k) \mathbf{H}_{i}(k) \mathbf{P}(k \mid k-1)$ are all also $p s d$. Moreover, from Eq. 18

$$
\begin{aligned}
\operatorname{det} \mathbf{P}(k \mid k)= & \operatorname{det}(\mathbf{P}(k \mid k-1) \\
& \left.-\mathbf{K}_{i}(k) \mathbf{H}_{i}(k) \mathbf{P}(k \mid k-1)\right)
\end{aligned}
$$

$$
\operatorname{det} \mathbf{P}(k \mid k) \leq \operatorname{det} \mathbf{P}(k \mid k-1)
$$

Substituting Eqs. 11 and 19 in Eq. 10 and solving for $\mathbf{P}_{f}$ we find that

$$
\mathbf{P}_{f}(k \mid k-1)=\mathbf{P}_{f}(k-1 \mid k-1)
$$

consequently

$$
\operatorname{det} \mathbf{P}_{f}(k \mid k) \leq \operatorname{det} \mathbf{P}_{f}(k-1 \mid k-1)
$$

The result in Eq. 20 shows intuitively how the a priori error covariance (before observations are made) is larger than the a posteriori (corrected) error covariance.

The final result on Eq. 23 does not hold however for the rest of the elements in Eq. 19. And in particular, to the newly introduced landmark quality covariance since it was formulated as possibly nonlinear and process noise dependent.

$$
\begin{aligned}
\mathbf{P}_{\mathcal{X}}(k \mid k-1)= & \nabla_{\mathcal{X}} \mathbf{f}_{\mathcal{X}} \mathbf{P}_{\mathcal{X}}(k-1 \mid k-1) \nabla_{\mathcal{X}} \mathbf{f}_{\mathcal{X}}^{\top} \\
& +\nabla_{\mathbf{v}} \mathbf{f}_{\mathcal{X}} \mathbf{Q}_{\mathcal{X}}(k) \nabla_{\mathbf{v}} \mathbf{f}_{\mathcal{X}}^{\top}
\end{aligned}
$$

\section{Case study: 3-dimensional visual landmarks for a planar robot}

In the case of the mobile robot platform used in these experiments the state variables included the pose of the robot on a plane, three-dimensional visual feature points for the landmarks, and scalar landmark quality states. The noisefree measurement equation for this configuration is

$$
\mathbf{h}_{i}(\mathbf{x}(k \mid k-1), 0)=\mathbf{R}^{\top}\left(\mathbf{x}_{f_{i}}-\mathbf{t}\right)
$$

with

$$
\begin{array}{r}
\mathbf{R}=\operatorname{Rot}(\theta(k \mid k-1), z) \\
\mathbf{x}_{f_{i}}=\left[x_{f_{i}}(k \mid k-1), y_{f_{i}}(k \mid k-1), z_{f_{i}}(k \mid k-1)\right]^{\top} \\
\left.\mathbf{t}=\left[x_{r}(k \mid k-1), y_{r}(k \mid k-1), 0\right)\right]^{\top}
\end{array}
$$

The measurement Jacobians in Eq. 17 for our planar mobile robot are computed with

$$
\begin{aligned}
\nabla_{r} \mathbf{h}_{i}(k) & =\left[\mathbf{0}_{3 \times 2} \mid \dot{\mathbf{R}}^{\top}\left(\mathbf{x}_{f_{i}}-\mathbf{t}\right)\right]-\mathbf{R}^{\top} \\
\nabla_{f_{i}} \mathbf{h}_{i}(k) & =\mathbf{R}^{\top} \\
\nabla_{\mathcal{X}_{i}} \mathbf{h}_{i}(k) & =\mathbf{0}
\end{aligned}
$$

and the measurement covariance $\mathbf{S}_{i}(k)$ is maintained as a running unbiased sample covariance of the form

$$
\mathbf{S}_{i}(k)=\frac{\left(n_{i}-2\right) \mathbf{S}_{i}(k-1)+\mathbf{e}_{i}(k) \mathbf{e}_{i}(k)^{\top}}{n_{i}-1}
$$




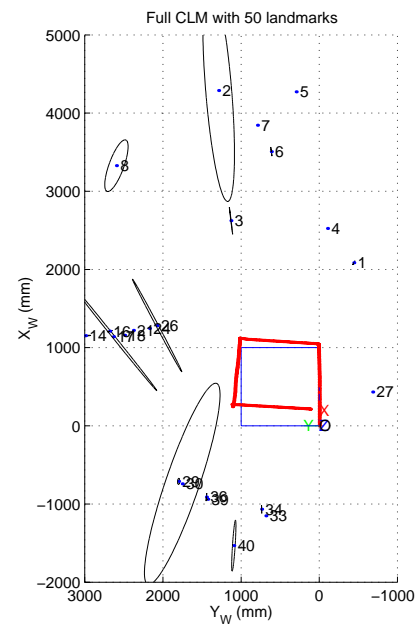

a) EKF-CML

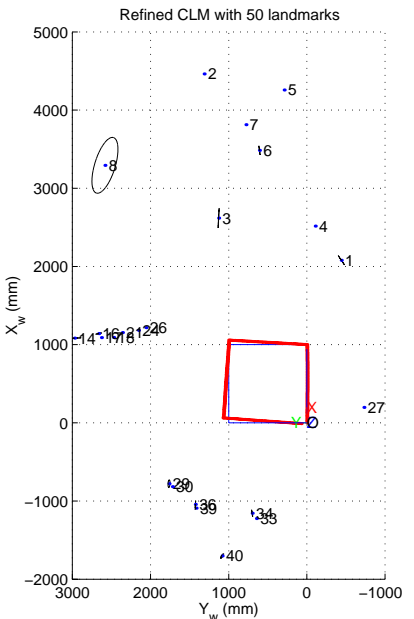

b) EKF-CML-LV
Figure 1. Concurrent localization and map building.

with $n_{i}$ a landmark identification accumulator.

Fig. 1 shows a run of the CML algorithm with and without landmark validation. The mobile robot was commanded along a squared trajectory and visual landmarks were automatically extracted with its vision system [1]. Those landmarks whose quality measure fell below a given threshold or whose sample covariance grew considerably were automatically reinitialized, resulting in a more accurate localization. Fig 2. contains a plot of the determinant of the map covariance for each landmark in the map. The figure exemplifies the result obtained in Sec. 6.

\section{Conclusions}

The main contribution of this work is the extension of the traditional full-correlation EKF CML algorithm for mobile robot localization and map building by adding landmark quality measures to the map state vector. These quality measures permit the maintenance of the map by the elimination of inconsistent observations. The proposed solution contributes in simplifying the data association problem in CML. Special attention has been paid in the selection of the process and observation models for landmark quality to guarantee that the uncertainty in the map estimates reduces monotonically.

\section{Acknowledgements}

This work was partially funded by the Spanish Council of Science and Technology under projects TAP98-0473 and DPI2000-1352-C02-01.

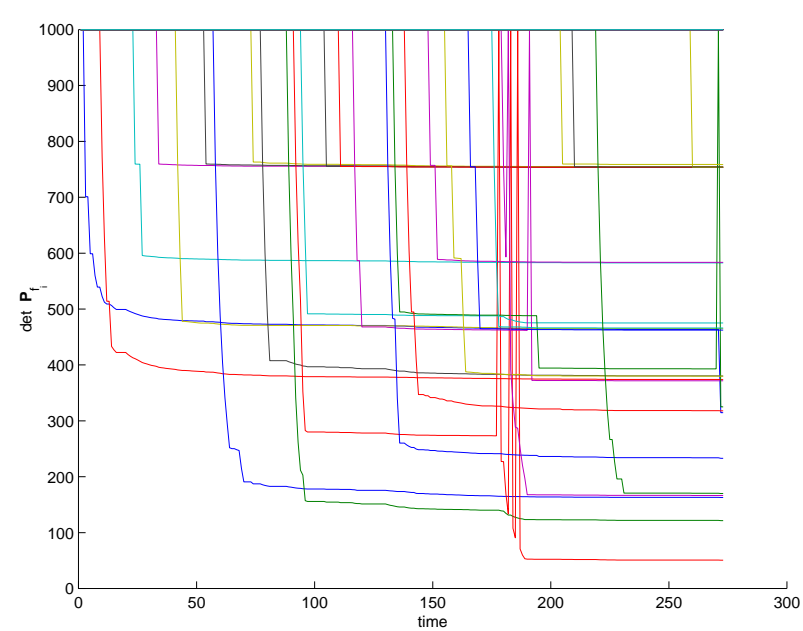

Figure 2. Determinant of the map covariance for each landmark.

\section{References}

[1] J. Andrade-Cetto and A. Sanfeliu. Learning of dynamic environments by a mobile robot from stereo cues. In Proc. IEEE Int. Conf. Multisensor Fusion Integration Intell. Syst., pages 305-310, Baden-Baden, Aug. 2001.

[2] J. A. Castellanos, J. M. M. Montiel, J. Neira, and J. D. Tardós. The SPMap: A probabilistic framework for simultaneous localization and map building. IEEE T. Robot. Automat., 15(5):948-952, Oct. 1999.

[3] A. J. Davison and N. Kita. Sequential localisation and mapbuilding for real-time computer vision and robotics. Robot. Auton. Syst., 36:171-183, 2001.

[4] M. W. M. G. Dissanayake, P. Newman, S. Clark, H. F. Durrant-Whyte, and M. Csorba. A solution to the simultaneous localization and map building (SLAM) problem. IEEE T. Robot. Automat., 17(3):229-241, Jun. 2001.

[5] D. Fox, W. Burgard, and S. Thrun. Markov localization for mobile robots in dynamic environments. J. Artif. Intell. Res., 30:391-427, Nov. 1999.

[6] J. J. Leonard, H. F. Durrant-Whyte, and I. J. Cox. Dynamic map building for an autonomous mobile robot. Int. J. Robot. Res., 11(4):286-292, 1992.

[7] J. J. Leonard and R. Rikoski. Incorporation of delayed decision making into stochastic mapping. In Proc. 7th Int. Sym. Experimental Robotics, Honolulu, Dec. 2000.

[8] P. M. Newman. On the Structure and Solution of the Simultaneous Localisation and Map Building Problem. PhD thesis, The University of Sydney, Sydney, Mar. 1999.

[9] R. C. Smith and P. Cheeseman. On the representation and estimation of spatial uncertainty. Int. J. Robot. Res., 5(4):5668,1986

[10] G. F. Welch. SCAAT: Incremental Tracking with Incomplete Information. PhD thesis, The University of North Carolina at Chapel Hill, Chapel Hill, Oct. 1996. 